\section{RF32 IDENTIFYING AN INTERVENTION DIFFERENTIAL EFFECT WITHIN HETEROSCEDASTIC LONGITUDINAL DATA - AN EXAMPLE USING CHILDHOOD GROWTH}

1,2,3 R Briscoe* 1,2,4 L Beggs, 1,5 C Griffiths, 1,2 GTH Ellison, 1,2 MS Gilthorpe. 'Leeds Institute for Data Analytics, University of Leeds, Leeds, UK' ${ }^{2}$ School of Medicine, University of Leeds, Leeds, UK; ${ }^{3}$ Leeds Teaching Hospitals Trust, Leeds, UK; ${ }^{4}$ National Institute for Health and Care Excellence, Manchester, UK; ${ }^{5}$ Sports Science, Leeds Beckett University, Leeds, UK

\subsection{6/jech-2018-SSMabstracts.120}

Background Because responses to interventions can be heterogeneous, interest may lie in the extent to which any given individual's response to an intervention relates to their baseline status (an intervention differential effect; IDE). In interventions designed to prevent excess childhood weight gain, researchers may want to investigate whether children with higher or lower body weights at baseline respond differently to the intervention. However, when investigating the potential of an IDE, it is necessary to avoid the issue of mathematical coupling (MC), where change in weight is analysed with respect to initial weight using correlation or regression. The problem of MC, and methods used to overcome it (Oldham's method and multilevel modelling) have been described previously for outcomes that are homoscedastic. However, the literature does not explore how these methods perform in identifying IDEs in outcomes that are inherently heteroscedastic (such as growth in childhood body weight). We hypothesised that methods for detecting IDEs in heteroscedastic outcomes are only robust when analyses in the intervention group are compared with analyses in control group data.

Methods We explored the performance of Oldham's method and multilevel modelling in overcoming MC within heteroscedastic data. We simulated longitudinal data derived from child weight growth statistics, designed to be heterogeneous to reflect real-world growth data. To emulate weight-management programmes, an intervention group was simulated with an IDE, and a control group was simulated without. Methods for detecting IDEs were evaluated: first in the intervention group only, then with analyses of the intervention group contrasted with the control group. Simulations were performed in $\mathrm{R}$ and MLwiN.

Results We demonstrated that Oldham's method and multilevel modelling were biased when used to estimate an IDE within inherently heteroscedastic data. However, we showed that introducing a control group comparison enabled both methods to robustly detect an IDE in heteroscedastic data, providing that parametric assumptions of growth were justified and modelled explicitly (e.g. as linear, quadratic, etc.).

Conclusion Oldham's method and multilevel models can robustly detect an IDE in growth data that are inherently heteroscedastic if analyses include a control group and underlying growth patterns can be parameterised appropriately. For study designs that do not collect control group data (as with most weight management programmes amongst children), identification of an IDE currently remains intractable.

\section{RF33 ARE ACTIVE BOYS AND GIRLS AT INCREASED RISK OF HOSPITAL ATTENDANCE OR ADMISSION FOR INJURY? A LONGITUDINAL STUDY IN WALES AND SCOTLAND USING LINKED COHORT AND ELECTRONIC HEALTH RECORDS}

${ }^{1} \mathrm{U}$ Griffiths*, 'M Cortina-Borja, ${ }^{2} \mathrm{~K}$ Tingay, ${ }^{2} \mathrm{~A}$ Bandyopadhyay, ${ }^{3} \mathrm{~A}$ Akbari, ${ }^{1} \mathrm{~B}$ DeStavola, ${ }^{1} \mathrm{H}$ Bedford, ${ }^{2} \mathrm{~S}$ Brophy, ${ }^{3} \mathrm{R}$ Lyons, ${ }^{4} \mathrm{C}$ Dezateux. ${ }^{1}$ Population, Policy and Practice Programme, UCL Great Ormond Street Institute of Child Health, London, UK; ${ }^{2}$ Swansea University Medical School, Swansea University, Swansea, UK; ${ }^{3}$ Farr Institute, Swansea University Medical School, Swansea University, Swansea, UK; ${ }^{4}$ Centre for Primary Care and Public Health, Barts and the London School of Medicine and Dentistry, Queen Mary University London, London, UK

\subsection{6/jech-2018-SSMabstracts.121}

Background Children and young people are being encouraged to increase the amount of time they spend being physically active, especially in activities of moderate and vigorous intensity. However, there is limited evidence on the prospective association of activity levels with injuries requiring health service utilisation. We examined the relationship between objectively-measured physical activity (PA) in childhood and subsequent hospital attendances or admissions for injuries, using linked electronic health records (EHR) from a nationally representative prospective cohort of children in Wales and Scotland. We hypothesised that children engaging in higher intensity $\mathrm{PA}$ at age seven experience higher rates of injuryrelated health service utilisation in later childhood and early adolescence.

Methods We analysed accelerometer-based estimates of moderate and vigorous (MVPA) and vigorous PA (VPA) from 1585 (777 [weighted \%: 46\%] boys) seven-year-old Millennium Cohort Study members, living in Wales or Scotland, whose parents consented to linkage of cohort to EHRs up until their 14th birthday. Negative binomial regression models adjusted by potential individual (sex, longstanding illness and weight status of child), household (poverty indicator, maternal age at birth of cohort child) and area-level (urban/ rural) confounders, allowed us to estimate associations between average daily minutes of MVPA, and VPA, and number of hospital in-patient admissions and emergency department attendances for injuries from age nine to 14 years.

Results Children spent a median of 59.5 (IQR 46.4, 75.2) and $18.1(12.5,25.3)$ minutes in MVPA and VPA/day respectively, with boys significantly more active than girls; $47.3 \%$ of children experienced at least one injury-related admission/attendance. Associations of each $10 \mathrm{~min}$ increment in MVPA and VPA with number of admissions/attendances were strong and significant in boys: adjusted rate ratio [RR] (95\% CI): 1.09 (1.01, 1.17) and $1.16(1.00,1.34)$, respectively, but not in girls: $0.94(0.86,1.03)$ and $0.85(0.69$, 1.04), respectively.

Discussion This is to our knowledge the first nationally representative prospective analysis examining associations between objectively measured PA in childhood and health service utilisation for injuries in childhood. We found that seven-year-old boys who engaged in more intense PA had higher injury-related admission or attendance rates from 
nine to 14 years than less active boys. The effects of MVPA and VPA in girls tended to be protective but were not significant. These findings may reflect gender differences in choice of active pursuits and will be the subject of future analyses.

\section{RF34 PARENT AND CHILD MISPERCEPTION OF CHILD WEIGHT STATUS: A CROSS-SECTIONAL ANALYSIS OF THE CORK CHILDREN'S LIFESTYLE STUDY (CCLAS)}

${ }^{1}$ E Kelleher, 'S Millar*, ${ }^{1,2}{ }^{2}$ Shiely, ${ }^{1} \mathrm{IJ}$ Perry, ${ }^{1} \mathrm{JM}$ Harrington. 'SChool of Public Health, University College Cork, Cork, Ireland; ${ }^{2}$ HRB Clinical Research Facility, Mercy University Hospital, Cork, Ireland

\subsection{6/jech-2018-SSMabstracts.122}

Background Despite the increased global awareness of childhood obesity, a high proportion of parents and children continue to misclassify child weight status. The aim of this study was to determine parent and child misperception of child weight and identify the determinants influencing this misperception.

Methods A cross-sectional study involving 1075 children, aged 8-11 years, drawn from primary schools in Cork city and county in Ireland. Data were collected using child and parent self-administered questionnaires. Physical measurements were taken by trained researchers according to standard procedures. Univariate and multivariable logistic regression analysis was used to examine factors influencing parental and child perceptions regarding child weight.

Results Almost one-quarter of parents of all children misclassified their child's weight status. Forty four per cent of parents of overweight or obese children underestimated their child's weight. In all children, factors associated with parental misperception of child weight included the child being female $(\mathrm{OR}=1.95 ; 95 \% \mathrm{CI} 1.36$ to $2.81, \mathrm{p}<0.001)$, being overweight or obese $(\mathrm{OR}=2.84 ; 95 \% \mathrm{CI} 1.95$ to $4.15, \mathrm{p}<0.001)$, child misclassification of own weight $(\mathrm{OR}=3.28$; 95\% CI 2.26 to 4.78, $\mathrm{p}<0.001)$ and parent reported child computer use $(\mathrm{OR}=1.64 ; 95 \% \mathrm{CI} 1.12$ to $2.39, \mathrm{p}=0.01)$. In overweight or obese children, accuracy in parental perception of weight improved with increasing child age $(\mathrm{OR}=0.49$; 95\% CI 0.27 to $0.88, \mathrm{p}=0.02)$. Of children who were overweight/obese, $76 \%(n=213)$ underestimated their weight. These children had increased odds of misperceiving their own weight status if their parents misclassified their child's weight $(\mathrm{OR}=3.98$; 95\% CI 1.95 to $8.10, \mathrm{p}<0.001)$.

Conclusion Findings suggest that in an obesogenic society, where overweight and obesity has become the norm, the capacity of both parents and children to correctly classify child weight status is significantly impaired. Health care professionals should be aware of the frequent misperception of weight status, especially when dealing with parents of younger children and children who are overweight or obese.

\section{RF35 EXAMINING ASSOCIATIONS BETWEEN ETHNIC DENSITY AND PHYSICAL ACTIVITY IN ADOLESCENTS: EVIDENCE FROM THE ORIEL STUDY}

N Berger*, D Lewis, S Cummins. Department of Public Health, Environments and Society, London School of Hygiene and Tropical Medicine, London, UK

10.1136/jech-2018-SSMabstracts. 123
Background Most adolescents do not achieve the recommended level of physical activity (PA) in the UK. The ethnic density hypothesis suggests that living in areas with higher proportions of people of the same ethnicity may confer health benefits. We explore whether school and neighbourhood ethnic densities are associated with adolescent utilitarian and leisure physical activity behaviours and whether these effects contribute to explaining ethnic differences in these behaviours.

Methods We used longitudinal data from the Olympic Regeneration in East London (ORiEL) study. In 2012, 3088 adolescents aged 11-12 were recruited from 25 schools in four deprived and ethnically diverse boroughs of East London. Adolescents were followed-up in 2013 and 2014. Own-group ethnic density was operationalised at school- and neighbourhood-levels in 2012/14 and 2011 respectively, and calculated as the percentage of adolescents, in the relevant school or neighbourhood (defined as 'lower layer super output area' of their home-address), who were of the same ethnic group. Analyses were restricted to White UK, White Mixed, Black African and Bangladeshi groups. The outcomes were selfreported binary variables: walking to school, walking for leisure, and outdoor PA. We identified potential confounders. We estimated logistic regression models with generalised estimating equations for each outcome and included interaction terms between ethnicity and ethnic densities. Item nonresponse was handled using multilevel multiple imputation. Final sample size was 1160 adolescents.

Results At school-level, there was consistent evidence that ethnic density amplifies ethnic differences in walking to school. For each 10 percentage point increase in ethnic density, there was evidence of increased probability of walking to school in Bangladeshi adolescents (OR=1.20; 95\% CI 1.09 to 1.31$)$ and decreased probability of walking to school in Black African $(\mathrm{OR}=0.58 ; 95 \% \mathrm{CI} 0.45$ to 0.75$)$ and White Mixed adolescents $(\mathrm{OR}=0.51 ; 95 \% \mathrm{CI} 0.35$ to 0.76$)$, after adjusting for confounders. Associations with walking for leisure and outdoor PA were in expected directions but not consistently observed in all ethnic groups.

At neighbourhood-level, evidence was more restricted. Amplification of ethnic differences was found for walking to school in Bangladeshi adolescents $(\mathrm{OR}=1.31 ; 95 \%$ CI 1.14 to 1.51) and for outdoor PA in White UK adolescents $(\mathrm{OR}=0.85 ; 95 \%$ CI 0.76 to 0.94$)$.

Conclusion These results suggest that ethnic density contributes to explaining differences in PA by amplifying ethnic differences in some forms of PA. Further research is needed to confirm these results in different populations and for different health behaviours.

\section{RF36 A CROSS-COUNTRY COMPARISON OF SELF-REPORTED EXPOSURE TO SUGARY DRINK MARKETING AND SUGAR-SWEETENED BEVERAGE INTAKE}

${ }^{1} \mathrm{H}$ Forde*, ${ }^{1} \mathrm{~J}$ Adams, ${ }^{1} \mathrm{M}$ White, ${ }^{2} \mathrm{~L}$ Levy, ${ }^{2} \mathrm{~F}$ Greaves. ${ }^{1} \mathrm{CEDAR}$, MRC Epidemiology Unit, University of Cambridge, Cambridge, UK; ${ }^{2}$ Public Health England, London, UK

\subsection{6/jech-2018-SSMabstracts. 124}

Background High sugar consumption contributes to the global obesity epidemic. Sugar-sweetened beverages (SSBs) constitute a significant proportion of sugar consumption and are independently associated with non-communicable diseases. Some 